\section{Ten weeks of home-based exercise attenuates symptoms of chemotherapy-induced peripheral neuropathy in breast cancer patients}

\author{
Karen Y. Wonders, 1,2 Gabrielle Whisler, \\ Hallie Loy, 2 Brian Holt,2 \\ Kelsey Bohachek, ${ }^{2}$ Robert Wise² \\ 1Department of Kinesiology and Health, \\ Wright State University, Dayton, OH; \\ 2Maple Tree Cancer Alliance, Dayton, $\mathrm{OH}$, \\ USA
}

\section{Abstract}

The purpose of this investigation was to determine if a structured, home-based exercise program was beneficial to reduce symptoms of chemotherapy-induced peripheral neuropathy and improve quality of life (QOL). A total of 50 women who are breast cancer survivors and are listed in the Breast Cancer Registry of Greater Cincinnati database were recruited by mail. Participants were initially asked to complete the McGill QOL questionnaire and the Leeds Assessment of Neuropathic Symptoms and Signs, before beginning a 10-week home-based exercise program. At the completion of the exercise program, subjects were asked again to complete the same two questionnaires. Pre- and postintervention data were analyzed using a repeated measures ANOVA, at a significance level of $\alpha<0.05$. Six individuals completed the investigation. Prior to the 10 -week exercise program, participants described their pain as unpleasant skin sensations (Pre-HBEx, $\mathrm{N}=6$ ), abnormally sensitive to touch (Pre-HBEx, $\mathrm{N}=6$ ), and coming on suddenly in bursts for no apparent reason (Pre-HBEx, N=5). Following 10 -weeks of exercise, participants reported experiencing less of these symptoms (PostHBEx, $\mathrm{N}=3,1$, and 4 respectively; $\mathrm{P}=0.05$ ). It was also determined that troublesome symptoms were significantly reduced after 10 weeks of home-based exercise $(\mathrm{P}=0.05)$.

\section{Introduction}

At present, more than 11.4 million men and women are living as cancer survivors; 1 a substantial increase from just a few decades ago. This surge in survivorship is primarily due to early detection and advances in treatment options. ${ }^{2}$ However, such treatments often result in long-term physical and psychological sequelae that impact the cancer survivor's quality of life. ${ }^{3}$ One such treatment option, chemotherapy, works by interrupting the cell cycle and preventing cell proliferation. ${ }^{4}$ While effective at stopping cancer growth, the negative side of systemic chemotherapeutic agents is that normal cells, as well as malignant cells, are disrupted. This leads to many side effects and often long-term morbidities, ${ }^{4}$ impacting functional ability and quality of life. The most common neurological side effect of chemotherapy is damage to the peripheral nervous system, ${ }^{5}$ which is referred to as chemotherapyinduced peripheral neuropathy (CIPN). The peripheral toxicity involved with CIPN is specific to each chemotherapy drug class, and in most cases, appears to be dose and duration dependent. The chemotherapy agents Docetaxel, Paclitaxel, or Vinorelbine often result in symptoms of CIPN.

The mechanism behind CIPN is not fully understood, and therefore prophylactic and symptomatic treatments are largely ineffective. ${ }^{6}$ While some of these therapies provide modest improvements in neurological function, in most instances, these agents are associated with additional negative side effects for cancer patients, including cardiac conduction defects and increased chemotherapy resistance. ${ }^{7-9}$ Thus, other interventions that address the symptoms of CIPN should be considered. One intervention that has the potential of preventing or alleviating CIPN is exercise rehabilitation. Two decades of research support the link between exercise and improved treatment tolerance in cancer patients. ${ }^{10-17}$ These studies focus on such toxicities as the effect of exercise on cardiovascular toxicity, musculoskeletal damage, fatigue, and quality of life, to name a few. However, there have been no published clinical trials examining the role of exercise in preserving neurological function following chemotherapy. It is plausible that exercise would attenuate symptoms of CIPN, as both short term and long term exercise have been shown to have a local effect on peripheral nerves, inducing positive changes in both the vasculature and metabolic systems. ${ }^{18}$ Numerous studies on the effect of exercise in populations with diabetic peripheral neuropathy have also produced promising results, ${ }^{14-16}$ further supporting this hypothesis.

In a previous investigation, our lab surveyed the current exercise behaviors of individuals experiencing symptoms of CIPN. Only $15 \%$ of patients surveyed were currently meeting the recommended levels of physical activity. These patients reported a significantly higher quality of life (QOL) and experienced less pain than their sedentary counterparts, ${ }^{19}$ lending some credibility to the hypothesis that an exercise intervention would be beneficial in attenuating symptoms of CIPN and improving the overall QOL of cancer patients. Thus, the purpose
Correspondence: Karen Y. Wonders, Department of Kinesiology and Health, Wright State University, 316 Nutter Center, 3640 Colonel Glenn Hwy, Dayton, OH 45435, USA.

Tel. +1.937.775.2637 - Fax: +1.937.775.4252.

E-mail: karen.wonders@wright.edu

Key words: home exercise, chemotherapyinduced peripheral neuropathy, breast cancer.

Conflict of interests: the authors report no conflict of interests.

Contributions: the authors contributed equally.

Conflict of interests: the authors declare no potential conflict of interests.

Received for publication: 13 March 2013.

Revision received: 1 May 2013

Accepted for publication: 6 May 2013.

This work is licensed under a Creative Commons Attribution NonCommercial 3.0 License (CC BYNC 3.0).

(C) Copyright K.Y. Wonders et al., 2013

Licensee PAGEPress, Italy

Health Psychology Research 2013; 1:e28

doi:10.4082/hpr.2013.e28

of this investigation was to determine if a structured, home-based exercise program is beneficial to reduce symptoms of CIPN and improve QOL.

\section{Materials and Methods}

\section{Subjects}

This investigation used a nonprobability sample of convenience. Women who met the eligibility criteria and were listed in the Breast Cancer Registry of Greater Cincinnati (BCRGC) database were recruited by mail. The eligibility criteria included women who i) have been diagnosed with CIPN by their oncologist, OR ii) were currently taking the chemotherapy agents Docetaxel, Paclitaxel, or Vinorelbine, iii) were previously sedentary, iv) have access to a computer and internet, and v) were able to read and write English. The BCRGC is a database established by the University of Cincinnati, Department of Environmental Health for the purpose of supporting studies that focus on breast cancer issues. Women who are breast cancer survivors and are located in the Greater Cincinnati Area may become members by signing a consent form and completing a self-report registration form that includes questions specific to the detection, diagnosis, and treatment of their disease. Approximately 50 women listed in the BCRGC 
met the eligibility criteria of the study and were recruited as study participants. All forms and procedures were approved by the Wright State University Institutional Review Board, as well as the BCRGC, prior to the onset of data collection.

\section{Data collection}

Data collection took place over three phases.

First phase: After a woman indicated her willingness to participate in the study, a packet was mailed to her. This packet contained an informed consent, a cancer history questionnaire, the McGill QOL questionnaire, the Leeds Assessment of Neuropathic Symptoms and Signs, and a self-addressed, stamped envelope. Women were asked to return the completed informed consent and questionnaires within two weeks of receiving the packet. Women who did not return the packet within two weeks were sent a reminder postcard.

Second phase: Once the packet was returned, a personal meeting was arranged between the PI and each woman. At this meeting, each woman was given a moderate-intensity home-based exercise program, along with three exercise thereabands (each of a different resistance), and a pedometer. Patients were given detailed instructions on how to perform each exercise listed on the form at a moderate intensity, monitor their heart rate, and stretch before and after each exercise. Also at this meeting, the patient was registered in the website www.onlinefitnesslog.com. This interactive website provides the opportunity for each patient to report and track her exercise status. After logging into the website, a detailed log sheet is provided to each patient, whereby she can enter such information as exercise duration, steps taken, sets and reps completed, rating of perceived exertion, and the like. The patient was instructed on how to log into the on-line program and fill out the activity log. Women were instructed to perform the home-based exercise program at a moderate-intensity (55-65\% of their estimated maximum heart rate), and their goals were increased gradually over the 10 week period. In Week 1 , the suggested goal was walking briskly twice/week for $10 \mathrm{~min}$, and by Week 10, walking for $30 \mathrm{~min} /$ day on at least 5 days during the week, consistent with the Surgeon General's recommendation. ${ }^{20}$ Participants were also instructed to do the resistance training exercises a minimum of 3 times each week for the 10 -week period. At the conclusion of each exercise session, women were asked to complete their activity log for monitoring of progress. Participants received weekly phone calls from the research staff, where the participant's activity was reviewed, progress was reinforced, and goals were set for the following week.

Third phase: At the completion of the 10week exercise period, a final packet was mailed to the patients. This packet contained the McGill QOL questionnaire and the Leeds Assessment of Neuropathic Symptoms and Signs. Patients were asked to fill out both questionnaires and send them back to the PI.

\section{Statistical analyses}

All results were expressed as means+SE. Pre- and post-intervention data (QOL and Neuropathic Pain) were analyzed using a repeated measures ANOVA. A significance level of $\alpha<0.05$ was used for all statistical analyses.

\section{Results}

\section{Subjects}

Initially, 50 women were contacted for participation in this investigation. A total of 14 women indicated a willingness to participate, however, 8 women dropped out of the study during the course of the 10 -week training program. Reasons for ceasing participation included lack of motivation to continue exercise program and being too busy to meet the exercise goals. Thus, a total of 6 females completed the 10-week home-based exercise program. Table 1 presents the subject characteristics of these participants, along with their course of treatment.

\section{Descriptive data and adherence}

Descriptive data for the continuous adherence measures were summarized. Sums of pedometer steps were calculated based on reported data. In the event of missing data (e.g. participant forgot to wear the pedometer while exercising), the total steps per week was based on the rest of her reported data. Table 2 presents Exercise Adherence Data. A significant increase in mean exercise session steps/week was observed from Week $1(4441.70 \pm 322)$ to Week $10(14673.43 \pm 4356)(\mathrm{P}<0.001)$.

\section{Psychological questionnaires}

The McGill QOL questionnaire was administered both before and after the 10-week home based exercise program. One question on the questionnaire was analyzed. Patients were asked to indicate on a scale of 1-10 the level of troublesome symptoms they have experienced related to their peripheral neuropathy over the last two days. Figure 1 presents the results from this analysis. It was determined that prior to the start of the exercise program patients reported significantly higher levels of troublesome symptoms (Pre-HBEx $=7.25 \pm 0.3$ ) than after 10-weeks of home-based exercise (PostHBEx $=3 \pm 0.4 ; \mathrm{P}=0.05$ ). Table 3 presents raw patient data, indicating each patient's response to this question on the QOL questionnaire.
Table 1. Subject characteristics.

\begin{tabular}{lc} 
Age (yrs \pm SE) & $51.6 \pm 2.6$ \\
Race & \\
$\quad$ Caucasian & 5 \\
African American & 1 \\
\hline Marital Status & \\
$\quad$ Single & 2 \\
$\quad$ Separated/divorced/widowed & 0 \\
$\quad$ Married & 4 \\
Educational level & \\
$\quad$ High school & 1 \\
$\quad$ Some college/college degree & 4 \\
$\quad$ Graduate school & 1 \\
\hline Current course of treatment & \\
$\quad$ Chemotherapy only & 3 \\
$\quad$ Radiation/chemotherapy & 5 \\
$\quad$ Surgery & 6 \\
\hline
\end{tabular}

Table 2. Exercise adherence data.

\begin{tabular}{lcc} 
& $\begin{array}{c}\text { Suggested } \\
\text { exercise } \\
\text { (min) }\end{array}$ & $\begin{array}{c}\text { Mean } \\
\text { pedometer } \\
\text { steps }\end{array}$ \\
Week 1 & 20 & 4441.7 \\
Week 2 & 30 & 6377.83 \\
\hline Week 3 & 40 & 7844.98 \\
Week 4 & 60 & 9005.66 \\
\hline Week 5 & 100 & 11555.87 \\
Week 6 & 100 & 11618.9 \\
\hline Week 7 & 125 & 13543.87 \\
Week 8 & 125 & 13243.44 \\
\hline Week 9 & 150 & 14568.94 \\
Week 10 & 150 & 14673.43 \\
\hline
\end{tabular}

Table 3. Raw patient quality of life data.

\begin{tabular}{lcc} 
Subject & Pre-EX & Post-EX \\
1 & 9 & 4.5 \\
2 & 9 & 3 \\
\hline 3 & 5.5 & 1 \\
4 & 6 & 6 \\
\hline 5 & 8 & 3 \\
6 & 6 & 1 \\
\hline Mean \pm SE & $7.25 \pm 0.3$ & $3.08 \pm 0.4$ \\
\hline
\end{tabular}

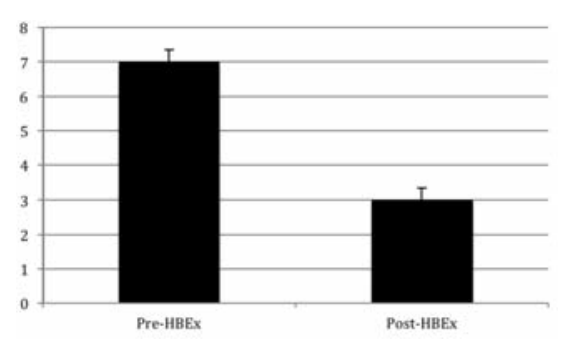

Figure 1. QOL Questionnaire. Level of troublesome symptoms related to peripheral neuropathy the patient has experienced over the last two days. Values are means \pm SE. $* P=0.05$ 
Table 4. Raw patient leeds assessment of neuropathic symptoms and signs questionnaire data.

\begin{tabular}{|c|c|c|c|c|c|c|}
\hline \multirow[b]{2}{*}{ Subject } & \multicolumn{2}{|c|}{ Unpleasant skin sensations } & \multicolumn{2}{|c|}{ Abnormally sensitive to touch } & \multicolumn{2}{|c|}{ Sudden bursts } \\
\hline & Pre-EX & Post-EX & Pre-EX & Post-EX & Pre-EX & Post-EX \\
\hline 1 & Y & Y & Y & Y & $\mathrm{Y}$ & Y \\
\hline 2 & $\mathrm{Y}$ & $\mathrm{N}$ & Y & $\mathrm{N}$ & Y & Y \\
\hline 3 & $\mathrm{Y}$ & $\mathrm{N}$ & Y & $\mathrm{N}$ & $\mathrm{Y}$ & $\mathrm{N}$ \\
\hline 4 & Y & $\mathrm{N}$ & Y & $\mathrm{N}$ & $\mathrm{N}$ & $\mathrm{N}$ \\
\hline 5 & Y & Y & Y & $\mathrm{N}$ & Y & Y \\
\hline 6 & Y & Y & Y & $\mathrm{N}$ & Y & Y \\
\hline Total yes & 6 & 3 & 6 & 1 & 5 & 4 \\
\hline
\end{tabular}

\section{Pain questionnaire}

The Leeds Assessment of Neuropathic Symptoms and Signs questionnaire asks respondents to classify and describe the neuropathic pain they experience. Prior to the 10week exercise program, participants described their pain as unpleasant skin sensations (PreHBEx, N=6), abnormally sensitive to touch (Pre-HBEx, N=6), and as coming on suddenly in bursts for no apparent reason (Pre-HBEx, $\mathrm{N}=5$ ). Following 10 -weeks of exercise, participants reported experiencing less of these symptoms (Post-HBEx, N=3, 1, and 4 respectively; $\mathrm{P}=0.05$ ). Table 4 presents raw patient data, indicating each patient's response to this question on the Leeds Assessment of Neuropathic Symptoms and Signs questionnaire.

\section{Discussion}

The purpose of this investigation was to determine if a structured, home-based exercise program was beneficial to reduce symptoms of CIPN and improve QOL. Major findings revealed that among those who completed the exercise program, 10-weeks of home-based exercise significantly lowered levels of both troublesome symptoms and pain symptoms (specifically, skin was less abnormally sensitive to touch). While pain sensations and sudden bursts of pain also appeared to decrease following the exercise program, these observations were not significant.

Resistance and aerobic exercise are associated with improvements in nerve conduction velocity 21,22 and increased axon regeneration. ${ }^{23}$ Long-term exercise has also been shown to induce changes in both the vasculature and metabolic systems. ${ }^{24}$ Chronic changes in blood flow can produce changes in the lumen diameter of blood vessels. Increased blood flow exposes blood vessels to shear stress, which augments vasodilation. ${ }^{25}$ Crosssectional studies have documented that regular, strenuous exercise results in larger lumen diameters than in untrained healthy con- trols. ${ }^{26}$ This vascular remodeling is robust and occurs even in response to moderate exercise training. ${ }^{27}$ This exercise-induced increase in capillarity works to offset the microvascular dysfunction which contributes to and exacerbates peripheral neuropathy. 28

In addition to these positive changes associated with the vasculature of the patient, it is possible that exercise may help decrease the pain associated with CIPN. In healthy subjects, acute exercise has been shown to transiently decrease pain perception; a condition referred to as Exercise-Induced Hypoalgesia (EIH). Both pain thresholds and pain tolerance levels have been reported to increase, while intensity ratings of pain appear to decrease following exercise intensities between 60 to $75 \%$ (up to $85 \%$ in women). ${ }^{29-31}$ Since the patients in the present investigation were instructed to exercise at a moderate intensity, it is plausible that they did not exercise at a high enough intensity to produce similar algesic effects.

\section{Conclusions}

Although this investigation produced promising results for individuals with CIPN, it was not without limitations. Namely, there was a low adherence rate. While the investigators tried to improve compliance through weekly phone calls and personal meetings, we had difficulty getting subjects to complete the 10week program. However, it is noteworthy that our adherence rates were similar to that of the healthy population, where approximately $50 \%$ drop out of an exercise program within the first 6 weeks of starting it. ${ }^{32}$ It is also plausible that the subjects did not exercise at a high enough intensity to produce more significant results. However, EIH has been reported following self-selected aerobic exercise intensities, in which the exercise intensity was determined by the subject. ${ }^{33,34}$ Since these exercise sessions were not supervised by a trained exercise professional, we have no way of knowing if the appropriate intensity was maintained throughout the exercise session, or if the patient over-reported their exercise intensity. Therefore future follow-up investigations involving an individualized exercise program prescribed and supervised by a certified Cancer Exercise Specialist are warranted.

Finally, the possibility of a placebo effect cannot be ruled out. Although there are no documented clinical trials examining the effect of exercise on symptoms of CIPN, it is plausible that if symptoms of CIPN become severe enough, they would prevent a patient from exercising all together. This could explain the high dropout rate noted in the present investigation, and could be an explanation for the high incidence of sedentary patients in our previous investigation.19

\section{References}

1. National Cancer Institute. Office of cancer survivorship. Available from: http:// dccps.nci.nih. gov/ocs/ researcher_ factsheet.pdf.

2. American Cancer Society. Cancer facts \& figures 2009. Atlanta, GA. Available from: http://www.cancer.org/acs/groups/content/@nho/documents/document/500809w ebpdf.pdf.

3. Ganz PA. Cancer survivorship: today and tomorrow. New York: Springer; 2007. pp 17.

4. Honea N, Brant J, Beck SL. Treatmentrelated symptom clusters. Sem Oncol Nurs 2007;23:142-51.

5. Quasthoff S, Hartung HP. Chemotherpayinduced peripheral neuropathy. J Neurol 2002;249:9-17.

6. Kaley TJ, DeAngelis LM. Therapy of chemotherapy-induced peripheral neuropathy. Br J Haematol 2009;145:3-14.

7. Goodman LS, Limbird LE, Milinoff PB, et al eds. Goodman and Gilman's: the pharmacological basis of therapeutics. 9th ed. New York: McGraw-Hill Professional; 1996.

8. Rao RD, Michalak JD, Sloan JA, et al. Efficacy of gabapentin in the management of chemotherapy-induced peripheral neu- 
ropathy. Cancer 2007;110:2110-8.

9. Suzuki T, Nishio K, Tanabe S. The MRP family and anticancer drug metabolism. Curr Drug Metab 2001;2:367-77.

10. Markes M, Brockow T, Resch KL. Exercise for women receiving adjuvant therapy for breast cancer. Cochrane Database Syst Rev 2006;4:CD005001.

11. Irwin ML, Crumley D, McTiernan A, et al. Physical activity levels before and after a diagnosis of breast carcinoma: the healthy eating, activity, and lifestyle (HEAL) study. Cancer 2003;97:1746-57.

12. Herrero F, San Juan AF, Fleck SJ, et al. Combined aerobic and resistance training in breast cancer survivors: a randomized, controlled pilot trial. Int $\mathrm{J}$ Sports Med 2005;26:294-302.

13. Aitken DR, James AG. Seromas and physiotherapy after mastectomy. Ann Surg Oncol 1997;4:293-7.

14. Schneider CM, Dennehy CA, Roozeboom M, Carter SD. A model program: exercise intervention for cancer rehabilitation. Integr Cancer Ther 2002;1:76-82.

15. Schneider CM, Hsieh CC, Sprod LK, et al. Effects of supervised exercise training on cardiopulmonary function and fatigue in breast cancer survivors during and after treatment. Cancer 2007;110:918-25.

16. Schneider CM, Hsieh CC, Sprod LK, et al. Exercise training manages cardiopulmonary function and fatigue during and following cancer treatment in male cancer survivors. Integr Cancer Ther 2007;6:23541.

17. Schneider CM, Hsieh CC, Sprod LK, et al. Cancer treatment-induced alterations in muscular fitness and quality of life: the role of exercise training. Ann Oncol 2007;18:1957-62.
18. Gustafsson T, Puntschart A, Kaijser L, et al. Exercise-induced expression of angiogenesis-related transcription and growth factors in human skeletal muscle. Am J Physiol 1999;276:H679-85.

19. Balducci S, Iacobellis G, Parisi L, et al. Exercise training can modify the natural history of diabetic peripheral neuropathy. J Diabetes Complications 2006;20:216-23.

20. Richardson J, Sandman D, Vela S. A focused exercise regimen improves clinical measures of balance in patients with peripheral neuropathy. Arch Phys Med Rehabil 2001;82:205-9.

21. Tesfaye S, Harris ND, Wilson RM, Ward JD. Exercise-induced conduction velocity increment: a marker of impaired peripheral nerve blood flow in diabetic neuropathy. Diabetologia 1992;35:155-9.

22. Wonders KY, Drury DG. Current exercise behaviors of breast cancer patients diagnosed with chemotherapy-induced peripheral neuropathy. J Integr Oncol 2012;1:1037.

23. Pate RR, Pratt M, Blair SN, et al. Physical activity and public health: a recommendation from the centers for disease control and prevention and the American College of Sports Medicine. U Am Med Assoc 1995;273:402-7.

24. Fisher MA, Langbein WE, Collins EG, et al. Physiological improvement with moderate in type II diabetic neuropathy. Electromyogr Clin Neurophysiol 2007;47:23-8.

25. Van Meeteren NL, Brakkee JH, Blessels GJ, et al. Effect of exercise training on acute (crush lesion) and chronic (diabetes mellitus) peripheral neuropathy in the rat. Restor Neurol Neurosci 1996;10:85-93.

26. Udina E, Puigdemasa A, Navarro X.
Passive and active exercise improved regeneration and muscle reinnervation after peripheral nerve injury in the rat. Muscle Nerve 2011;43:500-9.

27. Gustafsson T, Puntschart A, Kaijser L, et al. Exercise-induced expression of angiogenesis-related transcription and growth factors in human skeletal muscle. Am J Physiol 1999;276:H679-85.

28. Fukai T, Siegfried MR, Ushio-Fukai M. Regulation of the vascular extracellular superoxide dismutase by nitric oxide and exercise training. $\mathrm{J}$ Clin Invest 2000;105:1631-9.

29. Huonker M, Halle M, Keul J. Structural and functional adaptations of the cardiovascular system by training. Int J Sports Med 1996;17:S164-72.

30. Dinenno FA, Tanaka H, Monahan KD, et al. Regular endurance exercise induces expansive arterial remodeling in the trained limbs of healthy men. J Phys 2001;534:87-95.

31. Levy BI, Schiffrin EL, Mourad J, et al. Impaired tissue perfusion: a pathology common to hypertension, obesity, and diabetes mellitus. Circulation 2008;118:96876.

32. Koltyn KF, Wertz AL, Gardiner RL, et al. Perception of pain following aerobic exercise. Med Sci Sports Exerc 1996;28:141821.

33. Gurevich M, Kohn PM, Davis C. Exerciseinduced analgesia and the role of reactivity in pain sensitivity. J Sports Sci 1994;12:549-59.

34. Sternberg WF, Bokat C, Kass L, et al. Sexdependent components of the analgesia produced by athletic competition. Pain 2001;94:65-74. 